\title{
Comparisons of GPS/MET retrieved ionospheric electron density and ground based ionosonde data
}

\author{
L.-C. Tsai ${ }^{1,2}$, W. H. Tsai ${ }^{2}$, W. S. Schreiner ${ }^{3}$, F. T. Berkey ${ }^{4}$, and J. Y. Liu ${ }^{2,1}$ \\ ${ }^{1}$ Center for Space and Remote Sensing Research, National Central University (NCU), Chung-Li, Taiwan, R.O.C. \\ ${ }^{2}$ Institute of Space Science, NCU, Chung-Li, Taiwan, R.O.C. \\ ${ }^{3}$ University Corporation for Atmospheric Research, Boulder, Colorado, U.S.A. \\ ${ }^{4}$ Space Dynamics Laboratory, Utah State University, Logan, Utah, U.S.A.
}

(Received April 12, 2000; Revised November 20, 2000; Accepted December 21, 2000)

\begin{abstract}
The Global Positioning System/Meteorology (GPS/MET) mission has been the first experiment to use a low Earth orbiting (LEO) satellite (the MicroLab-1) to receive multi-channel Global Positioning System (GPS) carrier phase signals and demonstrate active limb sounding of the Earth's atmosphere and ionosphere by radio occultation technique. Under the assumption of spherical symmetry at the locality of the occultation, the dual-band phase data have been processed to yield ray-path bending angle profiles, which have then been used to yield profiles of refractive index via the Abel integral transform. The refractivity profiles can then, in turn, yield profiles of ionospheric electron density and other atmospheric variables such as neutral atmospheric density, pressure, and temperature in the stratosphere and upper troposphere, and water vapor in the lower troposphere with the aid of independent temperature data. To approach a near real-time process, electron density profiles can also be derived by the Abel transform through the computation of total electron content (TEC) assuming straight-line propagation (neglecting bending). In order to assess the accuracy of the GPS/MET ionospheric electron density retrievals, coincidences of ionosonde data with GPS/MET occultations have been examined. The retrieved electron density profiles from GPS/MET TEC observations have been compared with ionogram inversion results derived from digital ionospheric sounders operated by the National Central University (the Chung-Li digisonde; $24.6^{\circ} \mathrm{N}, 121.0^{\circ} \mathrm{E}$ ) and by Utah State University (the Bear-Lake dynasonde; $41.9^{\circ} \mathrm{N}, 111.4^{\circ} \mathrm{W}$ ). A fuzzy classification method for the automatic identification and scaling of ionogram traces has been applied to recorded ionograms, and then bottomside ionospheric electron density profiles are determined from true-height analysis. The comparison results show better agreement for both of the derived electron density profiles and the $F_{2}$-layer critical frequency $\left(f_{o} F_{2}\right)$ at midlatitude observations than at low-latitude observations. The $r m s f_{o} F_{2}$ differences from the GPS/MET retrievals are $0.61 \mathrm{MHz}$ to the Bear-Lake dynasonde measurements and $1.62 \mathrm{MHz}$ to the Chung-Li digisonde measurements.
\end{abstract}

\section{Introduction}

Since the mid-1960s, the radio occultation technique has been used to study the structure and properties of the atmospheres of Venus, Mars, some other outer planets, and many of their moons (Kliore et al., 1965; Lindal et al., 1979, 1981, 1987). As a spacecraft passed behind a planet, the radio paths of the signals would be systematically refracted by the vertical gradient of atmospheric refractivity; then the Doppler shifted signals are used to estimate atmospheric properties. With the completion of the Global Positioning System (GPS), the sensitivity and coverage necessary to improve upon existing data sets for the Earth's atmosphere in a compact, lowpower, and cost-effective manner are now available by GPS signal measurements. In 1993 the University Corporation for Atmospheric Research (UCAR) organized a proof-ofconcept experiment on a 735-km low Earth orbiting (LEO) satellite (the MicroLab-1 satellite) to receive GPS signals and demonstrate active limb sounding of the Earth's atmosphere

Copy right(C) The Society of Geomagnetism and Earth, Planetary and Space Sciences (SGEPSS); The Seismological Society of Japan; The Volcanological Society of Japan; The Geodetic Society of Japan; The Japanese Society for Planetary Sciences. and ionosphere by radio occultation technique. The main objectives of this GPS-LEO occultation mission were numerical weather prediction and long-term monitoring of the Earth's climate and thus it was termed the GPS/Meteorology (GPS/MET) program. GPS/MET has been able to sound the ionosphere and atmosphere from the orbit altitude to the Earth's surface, providing high-resolution profiles of ionospheric electron density, neutral gas density, temperature, and pressure. Conversely, if independent temperature profiles are given from other instruments or climatologies, one can use the derived refractivity values to compute the vertical distribution of water vapor (Zou et al., 1995; Ware et al., 1996; Kursinski et al., 1997; Rocken et al., 1997). These retrieval processes generally assume spherical symmetry of refractivity at the locality of occultations and use the Abel integral transform to invert the data. It is obvious that this assumption of spherical symmetry in the ionosphere is almost never true. Even so the range of science payoffs from occultation data is enormous, spanning disciplines from meteorology and climatology to ionospheric physics, space weather, and a suite of related earth science pursuits.

Another sources of ionospheric data that are analyzed in 
this paper are obtained from vertical incidence ionosondes. Although more sophisticated techniques for in-situ measurement of ionospheric parameters have since been developed, vertical incidence ionosonde still remains an inexpensive and viable environmental monitor at nearly every national Geophysical Observatory. The variation of the virtual height of reflection $h^{\prime}(f)$ as a function of the radio frequency is the fundamental ionosonde data product and the records of these measurements are known as ionograms. Synoptic ionospheric measurements derived from ionograms have provided valuable information both for high frequency (HF) propagation work and for studies related to the physics of the ionosphere. In this paper, an automatic ionogram scaling algorithm incorporating a fuzzy classification method (Tsai and Berkey, 2000) has been applied to obtain the virtual height-frequency trace and identify ionospheric parameters, including $f_{\min }, f_{o} E, f_{o} F_{1}, f_{o} F_{2}, h^{\prime} E, h^{\prime} E_{s}, h^{\prime} F, f_{x} I$, and $M(3000) F_{2}$. Even more information can be obtained from these fuzzy segmented records by inverting the virtual height-frequency trace to obtain an electron density profile, a process called true-height analysis. An efficient numerical integration method termed " $\mu$ ' $t$-fitting" (where $\mu$ ' is the group refractive index and $t$ is defined as $\sqrt{ }\left(1-f_{N}^{2} / f^{2}\right)$ for the O-waves and $\sqrt{ }\left[1-f_{N}^{2} /\left(f^{2}-f f_{H}\right)\right]$ for the X-waves $)$ is briefly described and can enable true height calculations to be readily carried out on small computer systems (Tsai et al., 1995). It can be used to analyze either the O- or X-echoes in an ionogram and includes the effect of the Earth's magnetic field.

The main purpose of this paper is to examine and estimate the accuracy of retrieved GPS/MET electron density profiles obtained with the Abel inversion by comparing them with vertical ionosonde data. It is noted that although GPSLEO occultation observation is horizontal UHF sounding with globally distributed snapshots and ionosonde is vertical/oblique HF sounding radar at one place, both systems are ionosphere (or/and atmosphere) delay measurements. Their first statistical comparison has been performed by Hajj and Romans (1998) and indicates that the fractional differences in $N_{m} F_{2}$ obtained from the GPS-LEO Abel inversion profiles and the nearby ionosonde data agree to about $\sim 20 \%$. In a latter work by Schreiner et al. (1999), the Abel inversion approach is validated in 4 days and 164 matches using a network of 45 ionosonde stations and agrees at a $13 \%$ $r m s$ level for the $f_{o} F_{2}$ correlative data and a $26 \% \mathrm{rms}$ level for the $N_{m} F_{2}$ correlative data. Recently, a more extensive comparison of 4411 occultations and the same 45 -ionosonde network data between April 1995 to February 1997 has been performed by the University Corporation for Atmospheric Research (UCAR) (Hajj et al., 2000). It has been noted that even though the differences are larger during the day in an absolute sense, the fractional difference is largest $(\sim 40 \%$ in $N_{m} F_{2}$ and $\sim 20 \%$ in $\left.f_{o} F_{2}\right)$ during the pre-dawn sector and smallest $\left(\sim 20 \%\right.$ in $N_{m} F_{2}$ and $\sim 10 \% f_{o} F_{2}$ ) around local noon. We should note that the 45 ionosonde stations are not uniformly distributed in global and only a few $(\sim 5)$ of those are located in the region of low latitude. In this paper, we will examine the results of $f_{o} F_{2}$ and electron density profiles from GPS/MET occultation observation using only two ground based modern ionosondes located at Chung-
Li, Taiwan $\left(24.6^{\circ} \mathrm{N}, 121.0^{\circ} \mathrm{E}\right)$ and Bear-Lake, Utah $\left(41.9^{\circ} \mathrm{N}\right.$ $\left.111.4^{\circ} \mathrm{W}\right)$. However, more correlative relations will be presented and discussed between $f_{o} F_{2}$ difference to latitude difference, longitude difference, and local time. We begin by considering the radio occultation problem and briefly describe the traditional Abel integral transformation technique used to inverse bending angle profile to ionospheric electron density profile. More description in Section 2 presents another Abel inversion technique through total electron content (TEC) data developed by Schreiner et al. (1999) and discuss the corresponding spatial coverage, resolution, and accuracy that can be expected for ionospheric electron density profiles derived from GPS-LEO occultation observations. Section 3 briefly describes the new automatic scaling algorithm based on a fuzzy segmentation and classification technique (Tsai and Berkey, 2000) to search the ionospheric reflected virtual-height versus plasma frequency trace, $h^{\prime}\left(f_{N}\right)$, and identify ionospheric parameters, e.g. $f_{o} E$ and $f_{o} F_{2}$. The traced results can be further processed by true-height analysis to obtain electron density profiles also. Section 4 shows both single occultation case and statistical comparisons of correlative data of the critical plasma frequencies obtained by both GPS/MET occultation observations and two ground based modern ionosondes located at Chung-Li, Taiwan, and Bear-Lake, Utah. Furthermore, the electron density profiles obtained from GPS/MET missions are also shown and compared with the ionogram inversion results.

\section{Derivation of Ionospheric Electron Density from Radio Occultation}

In the mid-1960's, a group at the Stanford University and the Jet Propulsion Laboratory introduced the Abel inversion technique (Fjeldbo and Eshelman, 1969; Fjeldbo et $a l ., 1971)$ to recover precise refractivity profiles from the observed bending angles induced by the atmosphere. Abel inversion has been the foundation of occultation analysis ever since. GPS/MET occultation observations are also made in a limb-scanning mode, where vertical scanning is provided by relative motion between the GPS and the LEO satellite as shown in the schematic representation of Fig. 1. In this paper we follow the inversion techniques suggested by Kursinski et al. (1997), Hajj and Romans (1998), and Schreiner et al. (1999), and evaluate it with real GPS/MET data. Theoretically, at microwave wavelengths in the Earth's ionosphere, the refractivity $N$ is a function of free electron density by the Appleton-Hartree equation (Budden, 1985). The effect is given to first order by

$$
N=(n-1) \times 10^{6}=-4.03 \times 10^{7} \frac{n_{e}}{f^{2}},
$$

where $n$ is refractive index, $n_{e}$ is electron number density per cubic meter, and $f$ is transmitter frequency in Hertz. The GPS satellites continuously transmit right-hand circularly polarized signals at two L band frequencies, $1.5754 \mathrm{GHz}$ (L1) and $1.2276 \mathrm{GHz}$ (L2). The uncertainty from higher order effect of the free electron density refractivity is less than $10^{-6}$ at two GPS sounding frequencies. Another uncertainty from the earth's magnetic field factor in the Appleton-Hartree equation is also less than $10^{-6}$ and can be ignored here. When a wave propagates in the low or middle atmosphere below 


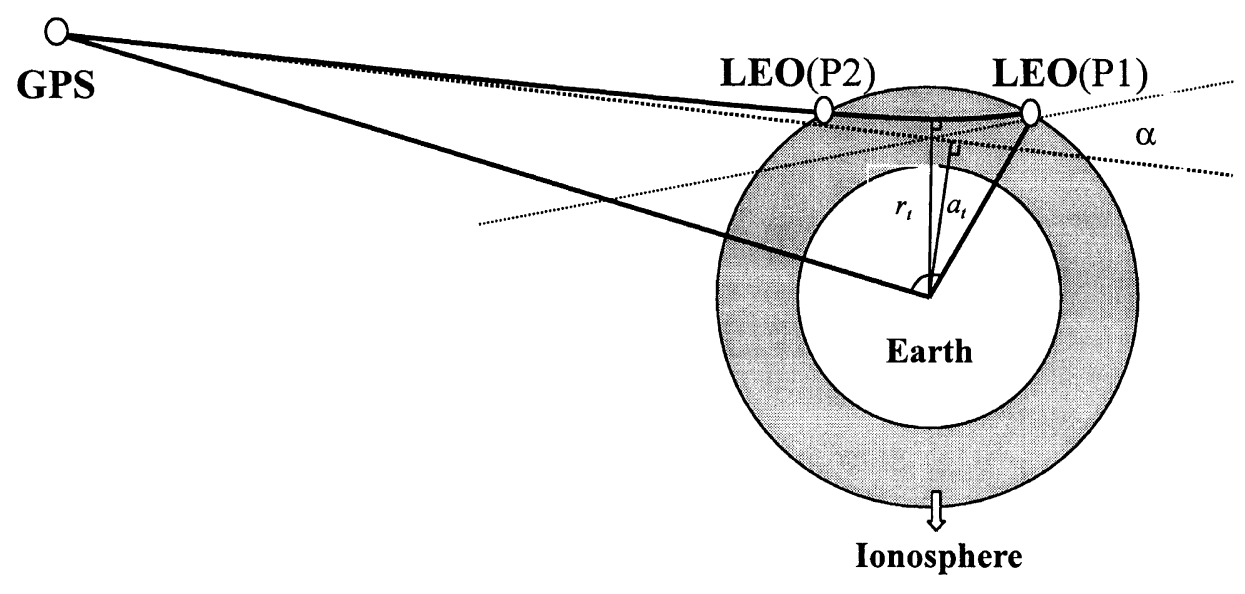

Fig. 1. Illustration of the geometry of the ray, bending angle $\alpha$, and impact parameter $a$ used in the GPS-LEO occultation problem for ionosphere observations.

$\sim 60-90 \mathrm{~km}, n$ contains more contributions from the other three main sources: the dry neutral atmosphere, water vapor, and particles of liquid water or ice crystal which are not discussed in this paper.

\subsection{Abel inversion of GPS-LEO bending angle profile}

In the geometrical optics approximation as shown in Fig. 1, a ray passing through the ionosphere is refracted according to Snell's law due to the vertical gradient of electron density and hence refractive index. The overall effect of the atmosphere can be characterized by a total bending angle $\alpha$, an asymptotic ray miss-distance or impact parameter $a$, and a tangent radius $r_{t}$ defined in Fig. 1. During an occultation, as the ray path descends or ascends through the atmosphere, the variation of $\alpha$ with the impact parameter $a$ is given by Snell's law when local spherical symmetry is assumed, and is expressed by

$$
\begin{aligned}
\alpha & =(a)=-\int_{r_{t}}^{r_{\mathrm{GPS}}} d \alpha+\int_{r_{t}}^{r_{\mathrm{LEO}}} d \alpha \\
& \approx-2 a \int_{r_{t}}^{\infty} \frac{1}{\sqrt{r^{2} n^{2}-a^{2}}} \frac{d \ln (n)}{d r} d r .
\end{aligned}
$$

As shown in the geometry of Fig. 1, a negative sign of the bending angle is such that the curvature of the ray is away from the center of the earth. In the case of a descending or setting occultation bending angle decreases from zero to a maximum negative value, and then increases to a positive value when the ray propagates through the lower atmosphere. The bending of the ray is directly proportional to the amount of phase rate change and usually changes sign from negative to positive at the point where the phase delay has its maximum magnitude near the $F_{2}$ peak. Using the Abel integral transformation (see Appendix) with the assumption of a spherically symmetrical atmosphere, the corresponding refractivity at a tangent radius $r_{t}$ can be expressed in terms of $\alpha(a)$ and the impact parameter $a$ as

$$
n\left(r_{t}\right) \approx \operatorname{Exp}\left(\frac{1}{\pi} \int_{a_{t}}^{\infty} \frac{\alpha(a)}{a^{2}-a_{t}^{2}} d a\right),
$$

where $a_{t}\left(=n\left(r_{t}\right) r_{t}\right)$ is the impact parameter for the ray whose tangent radius is $r_{t}$. The works of Hajj and Romans (1998) and Schreiner et al. (1999) provide more details of the calculation of bending angles and their corresponding impact parameter values from the observed excess Doppler shifts using the Bouguer's rule and occultation geometry properties. In this paper, electron density profiles have also been derived by the Abel transform but through the computation of TEC to approach a near real-time process as discussed in the next section.

\subsection{Abel inversion of GPS-LEO TEC profile}

Actually, even during the daytime and near solar maximum, the absolute magnitude of the bending angle does not exceed $0.03^{\circ}$ for both of L1 and L2 GPS frequencies in the $F$-region (Hajj and Romans, 1998; Schreiner et al., 1999). The ray separation from straight-line propagation can be easily estimated: $\Delta h \sim \alpha$ (in rad) $L_{R}$, where $L_{R}$ is the distance between tangent point and LEO position and is about 3100 $\mathrm{km}$ for the MicroLab-1 satellite in a $735-\mathrm{km}$ altitude orbit. This estimation shows that the height error is normally less than $2 \mathrm{~km}$, which is much smaller than typical vertical scales of the electron density in the ionosphere. Hence, if bending is neglected and spherical symmetry is assumed, then the Abel integral transform can be applied directly to derive ionospheric electron density from TEC data (Schreiner et al., 1999). We note that the straight-line assumption is not well applied to the case for $E$-layer ionosphere monitoring. However, the corresponding errors are much less than the inhomogeneous errors caused by the spherical symmetry assumption.

Formally the L1 and L2 signals propagation on different paths, and therefore the calculated TEC data on each path will be slightly different. However, because bending angles at GPS frequencies are negligible, then assuming straightline propagation is valid, and will allow the computation of TEC that is the same whether calculated from either $S_{1}$ or $S_{2}$, or from $S_{1}-S_{2}$. Therefore, the total electron content $T$ along a ray is related to electron density $n_{e}$, index of refraction $n$, and the excess phase $S_{1}$ or $S_{2}$ at either the L1 or L2 GPS carrier frequencies, respectively, and is given as

$$
\begin{aligned}
T & =\int n_{e} d l=-\frac{f^{2}}{40.3} \int(n-1) d l \\
& =-\frac{f_{1}^{2} S_{1}}{40.3}=-\frac{f_{2}^{2} S_{2}}{40.3}=\frac{\left(S_{1}-S_{2}\right) f_{1}^{2} f_{2}^{2}}{40.3\left(f_{1}^{2}-f_{2}^{2}\right)}
\end{aligned}
$$


where $T$ is in e/m $\mathrm{m}^{2}$, and $S_{1}$ and $S_{2}$ are in meters. It is noted that GPS is a one-way and code-based observing system, and orbit and clock errors induce GPS to be only a meterlevel ranging system directly from the carrier phase measurements. The use of dual GPS frequencies for TEC calculation can automatically remove both orbit and clock errors, and thus precise orbit determination and the related "doubledifferencing" techniques (Wu, 1984) with additional ground data for eliminating clock errors are not required, which allows inversion to be performed in near real time. We further define a "calibrated" TEC to be the TEC difference obtained from an occulting LEO position and another auxiliary LEO position having the same impact distance as illustrated as the $\mathrm{P} 1$ and $\mathrm{P} 2$ in Fig. 1. Under the assumption of local spherical symmetry, the calibrated TEC is also related to electron density through the following integral:

$$
\tilde{T}\left(r_{r}\right)=2 \int_{r_{t}}^{r_{\mathrm{LEO}}} \frac{r n_{e}(r)}{\sqrt{r^{2}-r_{t}^{2}}} d r,
$$

Applying the Abel transformation (see Appendix), as is similarly done with inversions through bending angles with an additional assumption of straight-line propagation, the electron density can then be given by the following integral equation.

$$
n_{e}(r)=-\frac{1}{\pi} \int_{r_{t}}^{r_{\mathrm{LEO}}} \frac{d \tilde{T}(r) / d r}{\sqrt{r^{2}-r_{t}^{2}}} d r .
$$

\subsection{Spatial resolution and accuracy analysis}

The spatial resolution of atmospheric profiles computed with radio occultation measurements has been discussed by Kursinski et al. (1997). Such vertical and horizontal resolutions can also be applied to ionospheric electron density profiles obtained by the Abel inversion through either bending angles or TEC computations. In GPS radio occultation experiments, because signals originate from a point source and are detected by a receiver phase-locked to the minimum phase delay, the cross-beam sampling at the limb takes the form of concentric Fresnel zones of a positive and negative response centered on the ray path. Vertical resolution is then limited by diffraction effects to the Fresnel zones. The contributions of adjacent Fresnel zones cancel so that the effective sampling can be represented by the first Fresnel zone (Born and Wolf, 1980). In the absence of a vertical refractivity gradient, i.e. ignoring ionospheric and significant atmospheric bending, the diameter of the first Fresnel zone $Z_{F}$ at the ray path tangent level is given by

$$
Z_{F}(\text { free space })=2\left(\frac{\lambda L_{T} L_{R}}{L_{T}+L_{R}}\right)^{0.5} \approx 2\left(\lambda L_{R}\right)^{0.5},
$$

where $\lambda$ is the GPS signal wavelength, and $L_{T}$ and $L_{R}$ are the distance from the transmitter and receiver to the limb respectively. $L_{T}$ is approximately $28500 \mathrm{~km}$ for the GPS transmitters and, for the MicroLab-1 satellite in a $735-\mathrm{km}$ altitude orbit, $L_{R}$ is about $3135 \mathrm{~km}$. Hence, at the GPS L1 wavelength and in the absence of radio bending, the vertical resolution $Z_{F}$ is about $1.4 \mathrm{~km}$. Meanwhile, diffractionlimited vertical resolution is also influenced by the vertical refractivity gradient. When this gradient increases, the vertical diameter of the first Fresnel zone decreases and vertical resolution improves in accordance with the expression

$$
\begin{aligned}
& \Delta Z=Z_{F}=Z_{F}(\text { free space }) M^{0.5}, \text { where } \\
& M=\left(1+L_{R} \frac{\partial \alpha}{\partial a}\right)^{-1},
\end{aligned}
$$

$\alpha$ is bending angle, and $a$ is the impact parameter. On the other hand, the horizontal resolution of GPS radio occultation observations can be defined by the distance traversed by the radio path as it enters and exits a layer with a vertical resolution of $\Delta Z$. In such case, the horizontal resolution $\Delta H$ and vertical resolution $\Delta Z$ are related by the approximate expression (Kursinski et al., 1997)

$$
\Delta H=2(2 R \Delta Z)^{0.5}
$$

where $R$ is the radius at the ray path tangent height. Thus the horizontal resolution of GPS-LEO occultation observation is about $280 \mathrm{~km}$.

Derived electron density precision is limited by the accuracy with which the GPS signal phases can be measured. However, in practice, inhomogeneous electron density in the horizontal direction for a given occultation is the main source of error in the Abel inversion. In fact, the ellipsoidal shape of the Earth and horizontal gradients in ionospheric structure produce small departures from spherical symmetry in the refractive index or electron density field used in the Abel inversion. Such errors are increased when limb sounding is propagating through the $E$-layer ionosphere and even the low and middle atmosphere. Other errors are caused by the assumption of straight-line propagation, which however can be ignoring in the height range from LEO altitude to bottom of the $F$ layer.

\section{Ionogram Analysis for Ionosonde Measure- ments}

Another sources of ionospheric data that are analyzed in this paper are vertical ionograms obtained from ionosonde measurements. First of all, to characterize and trace ionograms and scale ionospheric parameters, a method for the automatic identification of ionogram traces using fuzzy classification techniques was developed (Tsai and Berkey, 2000). Various fuzzy geometrical properties, including the topological concepts of connectedness, adjacency, height, width, and major axis, are used in ionogram analysis. Not only does the fuzzy segmentation process separate signals from the chaotic noise background that often characterizes ionograms, but it can also be applied to classify ionospheric echoes according to standard nomenclature, e.g. normal $E, F$, or $E_{s}$ layers. Furthermore, in reference to skeleton or thinning extraction in image processing, the fuzzy connectedness between echoes in selected segments can be used to determine the primary layers that are characteristic of vertical incidence ionospheric reflection for further scaling or true-height reduction.

Two examples of the fuzzy tracing method derived from Chung-Li and Bear-Lake ionograms are represented in the upper panels of Figs. 2 and 3, where color has been used to denote the various fuzzy segments identified by this method, while the black line indicates the resulting fuzzy traces. The quantitative reduction of ionograms to numerical values 


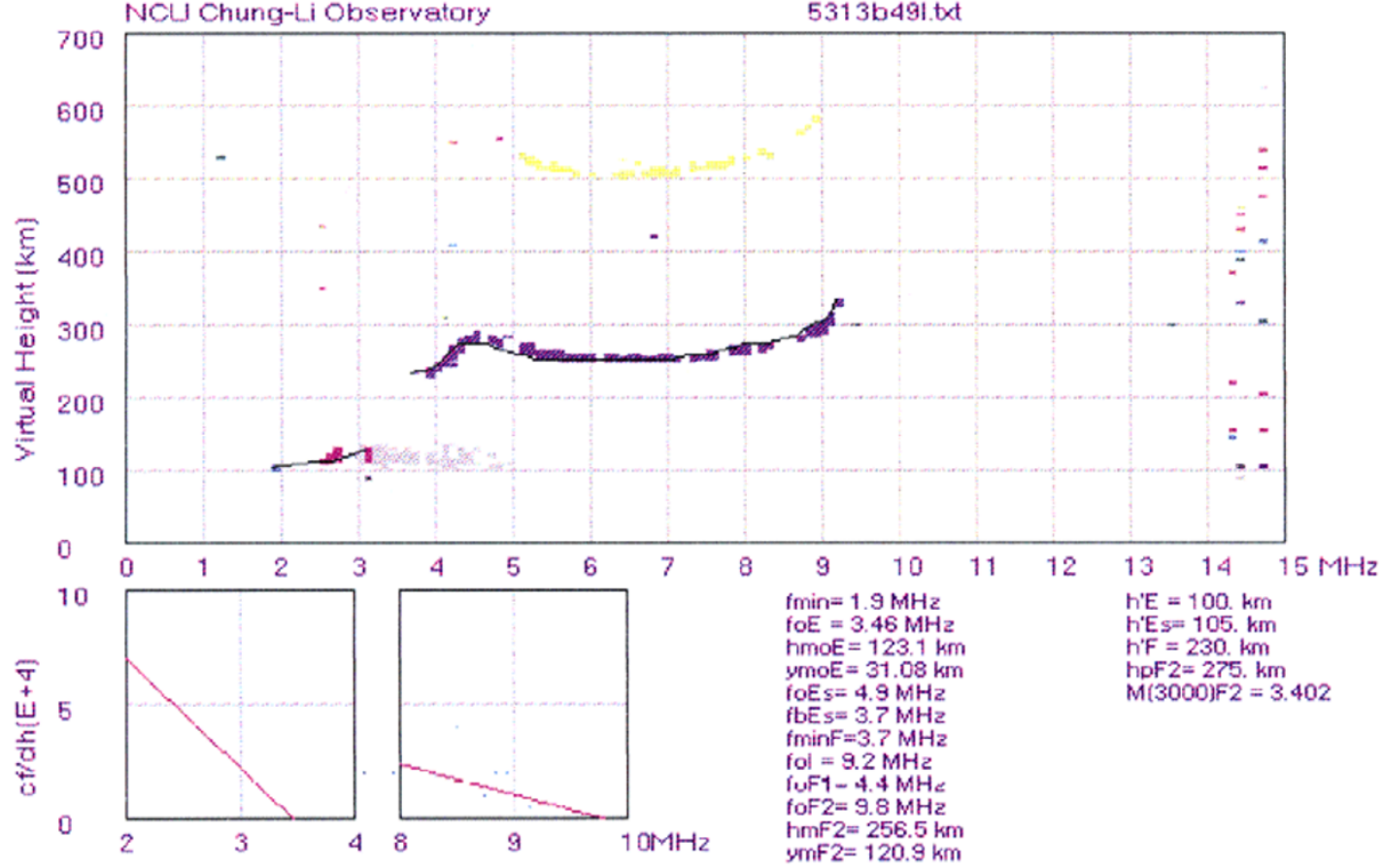

Fig. 2. The results of automatic trace identification and ionospheric parameter extraction are shown for ordinary mode echoes recorded by the Chung-Li digisonde. The upper panel shows the ionogram with different ionogram segments depicted in color; the black lines denote the ionospheric $E$ - and $F$-region traces as identifed by the fuzzy algorithm. The lower panels show how the fitting routine developed by Paul and Mackison (1981) is used to derive $f_{o} E$ and $f_{o} F_{2}$ for these data. The numerical information derived by the algorithm is shown in the lower left-hand corner of the diagram.

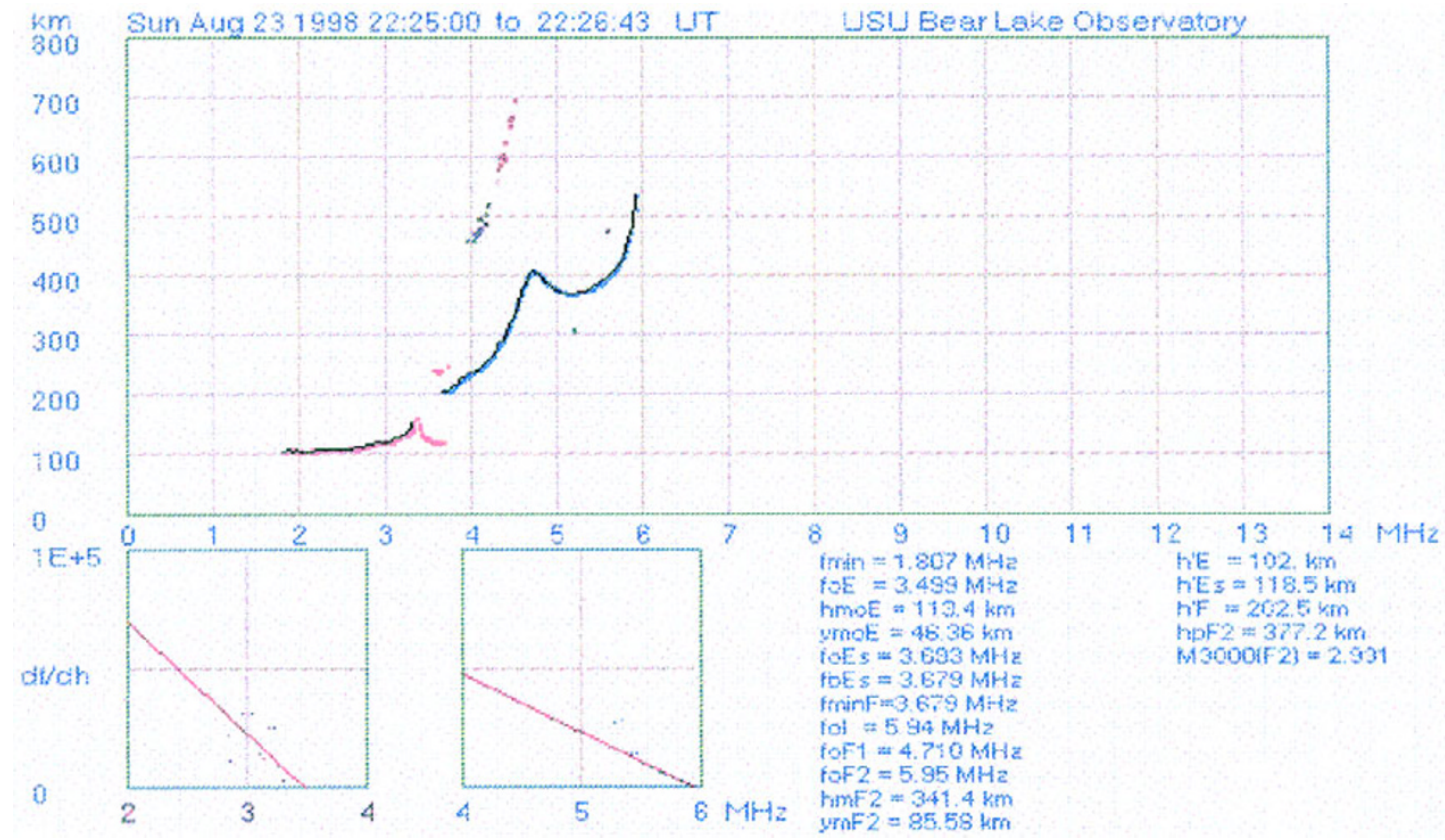

Fig. 3. Same as Fig. 1 for a daytime ionogram acquired with the USU Bear Lake dynasonde.

(commonly termed scaling) is intended to select certain features of the ionogram which are of particular significance for scientific or operational work. Following the U.R.S.I. standard, fourteen ionospheric parameters: $f_{\min }, f_{o} E, h^{\prime} E$, $f_{o} E_{s}, h^{\prime} E_{s}$, type of $E_{s}, f_{b} E_{s}, f_{o} F_{1}, M(3000) F_{1}, h^{\prime} F, h^{\prime} F_{2}$, $f_{o} F_{2}, f_{x} I$, and $M(3000) F_{2}$ have been identified as important geophysical parameters. In this paper, it is particularly important to obtain the critical frequency of the $F_{2}$ layer: $f_{o} F_{2}$, which will be calculated and compared to GPS/MET radio occultation observations. It is noted that while the echo classification and layer tracing procedures described here yield a frequency at the cusps of a layer that often does not represent 
the critical frequency but is called the "top" frequency of the layer. For our work, we have employed a method developed by Paul and Mackison (1981) to derive a more accurate estimate of the critical frequency; they denoted that when the radio frequency $f$ approaches the critical frequency $f_{c}$, the inverse of the slope of the virtual height versus radio frequency curve approaches zero linearly with the difference between $f$ and $f_{c}$. This behavior is still true in the general case when the Earth's magnetic field is included and the virtual heights are represented either by the ordinary or extraordinary mode. This principle can be used to extrapolate a very accurate estimate of the critical frequency. The bottom-left panels of Figs. 2 and 3 show the least-squares linear fitting for $d h^{\prime} / d f$ obtained from the fuzzy-linked traces and the extrapolation method which determines the critical frequencies for both the $E$ and $F$ layers. The annotation contained in Figs. 2 and 3 shows numerical results derived from our automated analysis procedures as applied to data obtained with the Chung-Li digisonde and the Bear-Lake dynasonde. The quality or accuracy of the autonomously scaled parameters can be determined from an examination of the data presented in these figures.

\section{1 “ $\mu^{\prime} t$-fitting” technique for true-height inversion}

Ionospheric sounding utilizes radio waves transmitted vertically from the ground, which travel upward into the ionosphere, where they are reflected, and return to a groundbased receiver. The measured virtual height $h^{\prime}$ (determined by signal time-of-flight) and the true height $h$ are related by $h^{\prime}=\int_{0}^{h_{r}} \mu^{\prime} d h$ theoretically, where $h_{r}$ is the height of the reflection, and $\mu^{\prime}$ is the group refractive index, a function of the radio frequency $f$, the plasma frequency $f_{N}$, the magnetic gyrofrequency $f_{H}$, and the magnetic dip angle $I$. Titheridge $(1985,1988)$ developed one of the most widely used trueheight analysis methods using polynomial equations to represent $h\left(f_{N}\right)$. The POLynomial ANalysis program (POLAN) is a computer method that implements a generalized, polynomial analysis, the execution time of which is primarily dependent on the group refractive index calculation. In this paper, we use another efficient numerical method to derive the integral of the group refractive index $\mu^{\prime}$, and enable trueheight analysis of digital ionograms to approach near realtime (Tsai et al., 1995). In order to avoid singular points in the integration of $\mu^{\prime}$, the product $\mu^{\prime} t$, where $t$ is defined as $\sqrt{ }\left(1-f_{N}^{2} / f^{2}\right)$ for the O-waves and $\sqrt{ }\left[1-f_{N}^{2} /\left(f^{2}-f \times f_{H}\right)\right]$ for the X-waves, has been approached by an interpolating fitting polynomial in $t$ and used in the true height calculations. We assume that the variation of plasma frequency $f_{N}$ with true height $h$ is given by

$$
h-h_{a}=\sum_{k=1}^{N_{T}} q_{k}\left(f_{N}^{2}-f_{a}^{2}\right)^{k},
$$

where the point $\left(f_{a}, h_{a}\right)$ is the origin for the real-height region under consideration, and the value of $N_{T}$ defines the order of the analysis. This model yields

$$
h^{\prime \prime}-h_{a}=\sum_{k=1}^{N_{T}}\left[2 q_{k} \int_{f_{a}}^{f_{R}} \mu^{\prime}\left(f_{N}^{2}-f_{a}^{2}\right)^{k-1} f_{n} d f_{N}\right],
$$

where $h^{\prime \prime}$ is the reduced virtual height, equal to $h^{\prime}$ minus the group retardation due to those parts of the profile below the point $\left(f_{a}, h_{a}\right)$. Therefore, by using the $\mu^{\prime} t$-fitting technique and replacing $f_{N}^{2}$ with $\phi$, we can express the integral in the above equation in terms of the known $\mu^{\prime} t$-fitting polynomial and improve the efficiency of the corresponding computation. Calculations normally proceed in a stepwise fashion to obtain successive, overlapping sections of the real-height profile. POLAN invokes a five- or twelve-term Gaussian quadrature integration, depending on the desired accuracy. For the parabolic lamination method $\left(N_{T}=2\right)$, the $\mu^{\prime} t$-fitting technique utilizes $38-62 \%$ fewer numerical operations than the Gaussian quadrature method.

\subsection{Accuracy analysis for true-height inversion}

In general, the interpolating polynomial does not produce results which are identical with the real product $\mu^{\prime} t$, and the non-matching values obtained from the interpolating polynomial are subject to error, which decreases (as expected) as the order of the interpolating polynomial is increased. From the works by Tsai et al. (1995), an accuracy test has been done for a model profile adopted by U.R.S.I. Working Group G.6.2 (McNamara and Titheridge, 1977), in which the testing profile consists of two overlapping Chapman layers, and the Chapman tail is cut off at $0.8 \mathrm{MHz}$. Virtual heights were calculated using a 40-point Gaussian integration applied over five successive height ranges, yielding errors of less than $1 \mathrm{~m}$. We have used the 5- and 12-term $\mu^{\prime} t$-fitting techniques to analyze 150 data points in which the frequency interval is $\geq 0.02 \mathrm{MHz}$ as recommended by McNamara and Titheridge (1977). The $r m s$ deviations of the different errors are 37.6 and 6.2 meters for the O-wave component using 5- and 12-term $\mu^{\prime} t$-fitting techniques, respectively, and 7.1 and 7.0 meters for the X-wave component. This result showed that, for the $\mathrm{O}$-wave component, application of the 12-term $\mu^{\prime} t$-fitting technique to the testing profile improves the difference error by a factor of $\sim 6$ smaller than that obtained with the 5 -term $\mu^{\prime} t$-fitting technique. However, for the X-wave component, the different error is essentially the same for each frequencyheight data pair when either the 5- and 12-term $\mu^{\prime} t$-fitting techniques is applied. This result is consistent with a smaller error in the interpolating fitting polynomial for the $\mu_{x}^{\prime} t$ curve than that derived for the $\mu_{o}^{\prime} t$ curve.

In principle, the true-height analysis error induced by the inversion numerical technique itself are very small and can usually be ignored comparing to other errors in the ionosonde measurement, e.g. virtual-height measurement precision, starting-height error, EF valley modeling error, and the error induced by gaps in virtual height information. The ionograms described here were recorded by modern digital ionosondes of the digisonde (Bibl and Reinisch, 1978; Reinisch, 1986) at Chung-Li, Taiwan, and the National Oceanic and Atmospheric Administration (NOAA) HF radar or termed the dynasonde (Grubb, 1979; Argo and Hindman, 1987) at Bear-Lake, Utah. The Chung-Li ionograms were stepped through the sounding frequency in increments of $100 \mathrm{kHz}$; the virtual height has 128 height ranges for a resolution of $5 \mathrm{~km}$. The Bear-Lake ionograms were acquired using the B-mode sounding pattern (Wright and Pitteway, 1979) and have a sounding frequency resolution less than 50 $\mathrm{kHz}$. The corresponding virtual-height resolution is close to the range sampling resolution, which is $0.75 \mathrm{~km}$ obtained from half of the product of the light speed (in free space) 
and a sampling interval of $5 \mu \mathrm{sec}$. Meanwhile, ionograms recorded by ground based ionosondes are absent of virtualheight records of $D$-layer reflection (in daytime) or $E$-layer reflection (in nighttime) due to strong ionospheric absorption and any region of decreased electron density in the ionosphere, e.g. $E F$ valley and the heights above the peak of the $F_{2}$ layer. This absent virtual height information, especially the low frequency reflection (the starting problem) and $E F$ valley reflection, leads to considerable uncertainties in the calculated electron density profile. Gulyaeva et al. (1978) suggested that it must be possible to use the differences of the ordinary and extraordinary components of ionograms to resolve the reflection absence problem. However, only on rare occasions is a complete ionogram trace of the extraordinary component available because of interference and larger ionospheric absorption for extraordinary waves. Other inversion techniques for the starting and valley problems assume standard models proposed by Titheridge $(1985,1986)$ for the POLAN program. In this paper, we used the suggested $E F$ valley model by Titheridge (1985) and a starting height obtained from associated GPS/MET retrieved electron density profile.

\section{Comparisons of GPS/MET Retrievals with Ground-Based Ionosondes}

In order to assess the accuracy of the GPS/MET Abel inversions, we compare the results of $f_{o} F_{2}$ estimates and electron density profiles from GPS-LEO occultation observations with a low-latitude digisonde located at Chung-Li, Taiwan $\left(24.6^{\circ} \mathrm{N}, 121.0^{\circ} \mathrm{E}\right)$, and a mid-latitude dynasonde located at Bear-Lake, Utah $\left(41.9^{\circ} \mathrm{N}, 111.4^{\circ} \mathrm{W}\right)$. We applied the Abel transform to calibrated TEC values derived by dual frequency excess phases; the excess phases were obtained from $0.1 \mathrm{~Hz}$ GPS carrier phase change data but interpolated using a cubic spline fit. Due to the orbit geometry of the MicroLab-1 satellite and the 24 GPS transmitters, only about 250 rising or setting occultations per day have been collected where the GPS-LEO paths are less than 45 degrees out of the MicroLab-1 orbit plane. For comparison with the Chung-Li digisonde, we collected 98 matches out of 152 occultations from 67 days (Oct. 10 to Nov. 1 and Nov. 24 to Dec. 15 in 1995, and Feb. 2 to Feb. 23 in 1997) where the location of the tangent point at the $F_{2}$ peak of each occultation are within \pm 15 degrees of the Chung-Li digisonde site in latitude and longitude. Most missing of the matches is caused by no operation of the Chung-Li digisonde. A few of missing of the matches (6 out of 152 occultations) is caused by strong $E_{s}$-layer reflection and then no $f_{o} F_{2}$ scaled. Figure 4 shows some examples of the LEO satellite position projections (onto a flat Earth) of these matched cases in colors for different occultation measurement. The larger solid square points represent the locations of the top tangent point for each occultation. Figure 5 shows the corresponding comparisons of derived occultation $f_{o} F_{2}$ (larger solid square points) with the Chung-Li digisonde $f_{o} F_{2}$ profiles (the dotted lines) for examples of Fig. 4 all as a function of local time. The $f_{o} F_{2}$ profiles from the Chung-Li digisonde were obtained at a sounding interval of 5 minutes and recorded within plus or minus one hour of the observing time at the top tangent point for each occultation. The corresponding latitude difference (shown by the letter $\mathrm{N}$ or $\mathrm{S}$ ) and the longitude difference (shown by the letter E or W), where N, S, E, and W mean north, south, east, and west, respectively, from the occultation tangent point at the $F_{2}$ peak to the Chung-Li digisonde site are also positioned in degrees as a function of local time and shown in the corresponding case color. The position differences can be used to check their association to the $f_{o} F_{2}$ differences.

More statistical comparisons and measures of association from these 98 matched observations are shown in Fig. 6. The left-upper panel in Fig. 6 illustrates a scatterplot and the corresponding least-squares fitting line for the GPS/MET $f_{o} F_{2}$ values versus the averaged $f_{o} F_{2}$ values of the associated digisonde measurements in \pm 1 hour of each occultation observing time. The results have a high linear correlation coefficient of 0.85 and a fitting line with a slope of 0.96 and a bias of $0.54 \mathrm{MHz}$. It is noted that the value of linear correlation coefficient lies between -1 and 1 , and a higher correlation coefficient magnitude equates to a higher positive (closed to +1 ) or negative (closed to -1 ) association between two variables. The corresponding $r m s f_{o} F_{2}$ difference is $1.62 \mathrm{MHz}$ or $23.5 \%$ in a fractional sense. Differences in these two measurements should be due to (1) errors due to the spherical symmetry assumption of the GPS/MET retrievals, (2) errors in the scaling analyses of ionograms, and (3) spatial and temporal mismatch. We next turn to measures of association between the $f_{o} F_{2}$ difference and other variables, e.g. local time and location distance. The rightupper panel, left-lower panel, and right-lower panels illustrate scatterplots and their least-squares fitting lines of the corresponding $f_{o} F_{2}$ differences in functions of local time, latitude difference in degree, and longitude difference in degree, respectively. It is noted that, in the left-lower panel of Fig. 6, there is a negative association with a linear correlation coefficient of -0.20 between the $f_{o} F_{2}$ difference and the latitude difference from the GPS/MET measurement to the ionosonde measurement. The fitting line's slope at -0.05 $\mathrm{MHz}$ per degree in latitude (with a bias of $-0.22 \mathrm{MHz}$ ) denotes higher latitude having lower critical frequency. More description for the association between derived $f_{o} F_{2}$ difference and latitude difference will be discussed more in the next analysis of the mid-latitude Bear-Lake dynasonde data. Furthermore, there is not much association of the $f_{o} F_{2}$ difference related to either local time (the right-upper panel) or the longitude difference (the right-lower panel) with linear correlation coefficients of -0.11 and 0.12 , respectively. However, from the scatterplot of the $f_{o} F_{2}$ difference versus local time, the $f_{o} F_{2}$ values by the GPS/MET occultation observations are underestimated at a ratio of $>75 \%$ during 12 AM to 8 PM (local time). Such results could be due to the equatorial anomaly. It is well known that the equatorial anomaly is caused by vertical electrodynamic drift at the equator and plasma diffusion along geomagnetic field lines in terms of a fountain effect (Martyn, 1959). When the equatorial anomaly is occurred, the daytime ionization density at the $F_{2}$ peak shows a pronounced trough at the magnetic equator and crest at about $20^{\circ} \mathrm{N}$ and $20^{\circ} \mathrm{S}$ magnetic dip. Furthermore, during years of sunspot minimum the equatorial anomaly is most pronounced at about $1400 \mathrm{LT}$ and then declines steadily until it disappears around 2000 LT (Rastogi, 1959). We note 


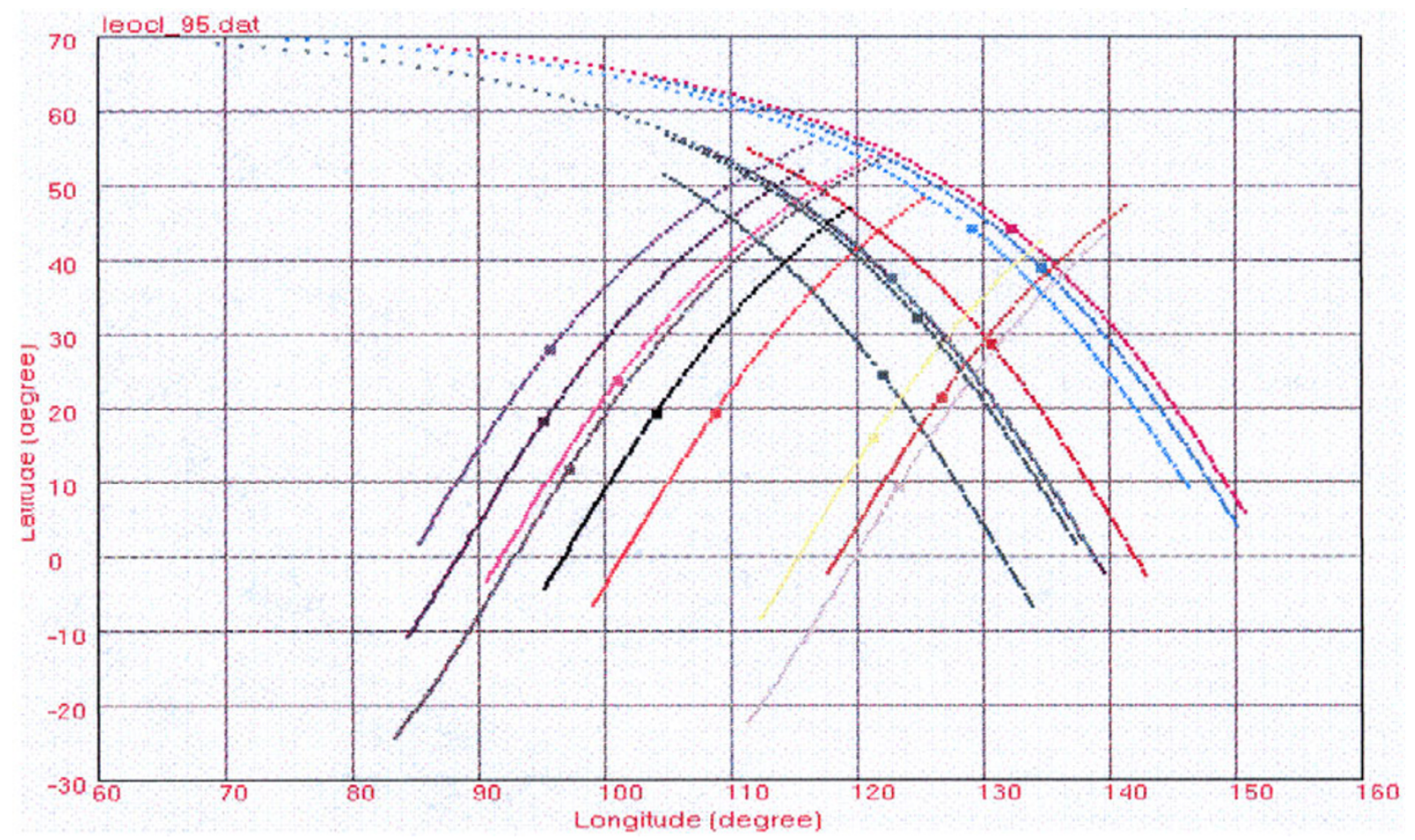

Fig. 4. Seventeen examples of LEO satellite position projections for GPS/MET occultation measurements above the Chung-Li digisonde site (24.6 ${ }^{\circ}$, $\left.121.0^{\circ} \mathrm{E}\right)$. The positions are projected onto a flat Earth under latitude vs. longitude coordinates and shown in different colors for different occultation measurements.

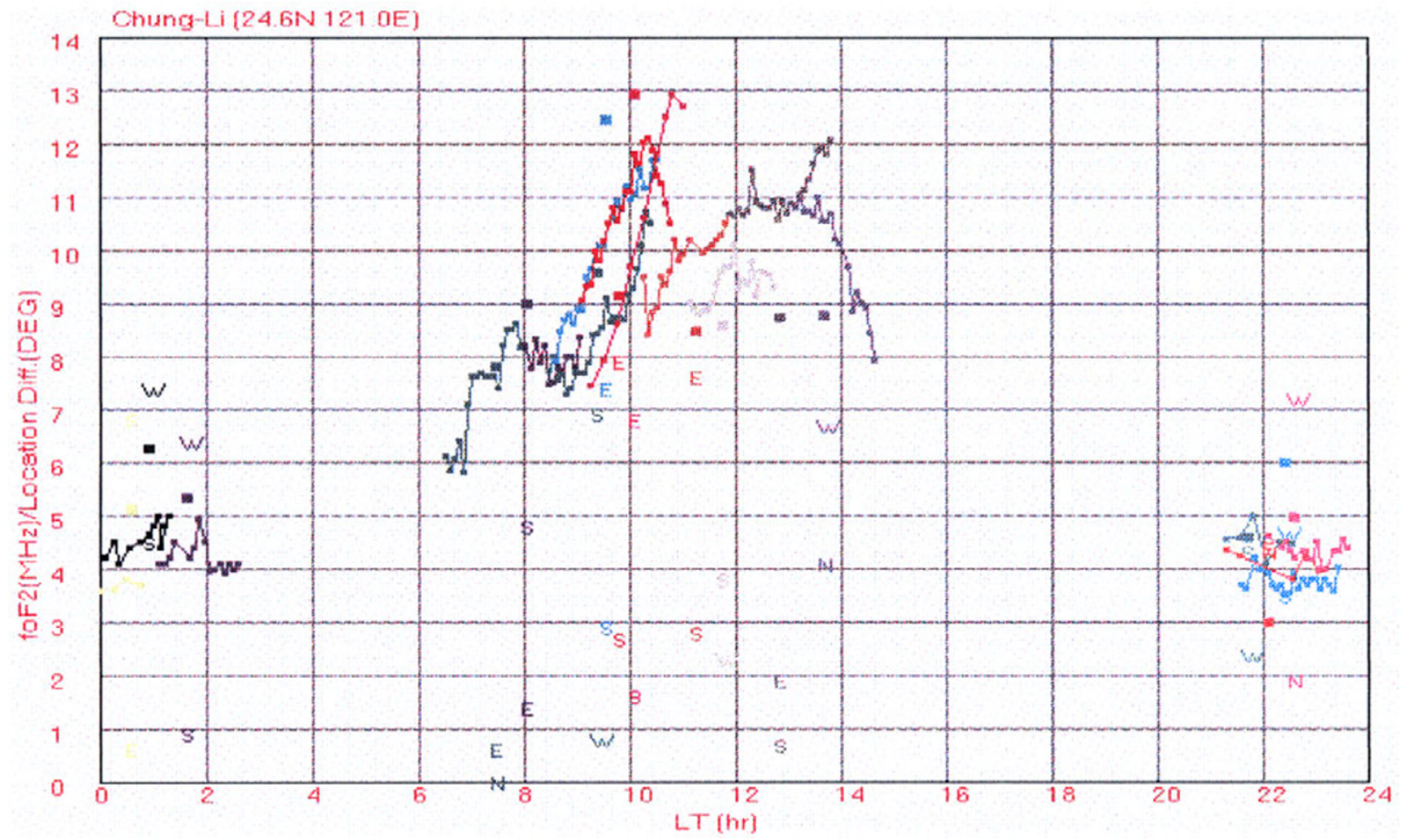

Fig. 5. Comparisons of 17 cases (the same as Fig. 4) of derived $f_{o} F_{2}$ s from GPS/MET occultation measurements (large solid square points) and Chung-Li digisonde observations (dotted line profiles) are shown in function of local time. Cases of occultation and concurrent digisonde measurements are shown in colors. The associated latitude difference (shown by letter $\mathrm{N}$ or $\mathrm{S}$ ) and longitude difference (shown by letter $\mathrm{E}$ or W) from the coordinate of the impact point with maximum electron density by GPS/MET occultation measurements to the Chung-Li digisonde site are positioned in degree and shown in the case color also. 

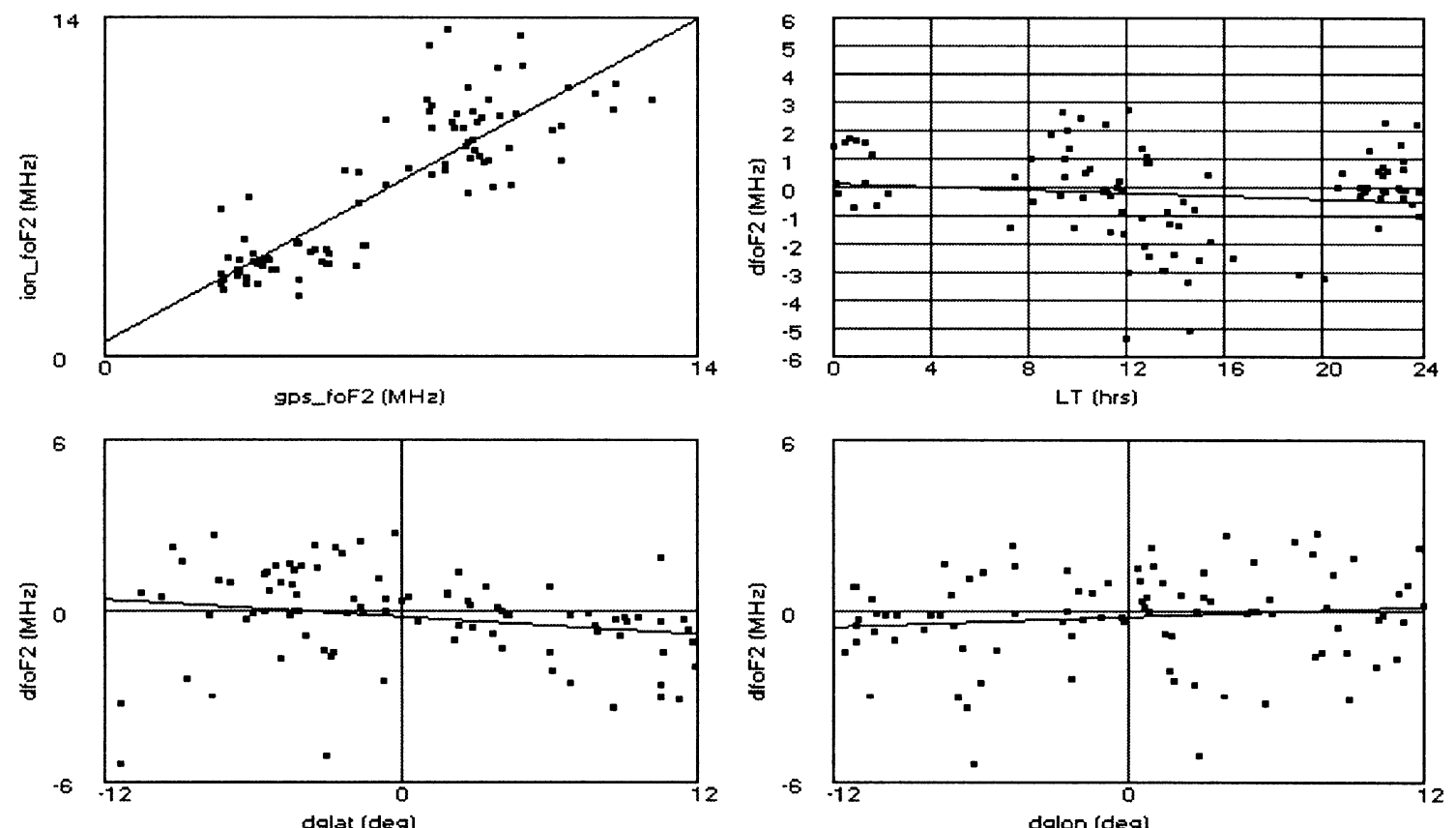

Fig. 6. The left-upper panel illustrates a scatterplot of $f_{o} F_{2}$ observed from GPS/MET occultation measurements and ionosonde measurements above Chung-Li $\left(24.6^{\circ} \mathrm{N}, 121.0^{\circ} \mathrm{E}\right)$, Taiwan. The right-upper panel, left-lower panel, and right-lower panels illustrate scatterplots of the associated $f_{o} F_{2}$ differences in functions of local time, latitude difference, and longitude difference, respectively. The associated linear correlative coefficients are 0.85 (left-upper panel), -0.11 (right-upper panel), -0.20 (left-lower panel), and 0.12 (right-lower panel).
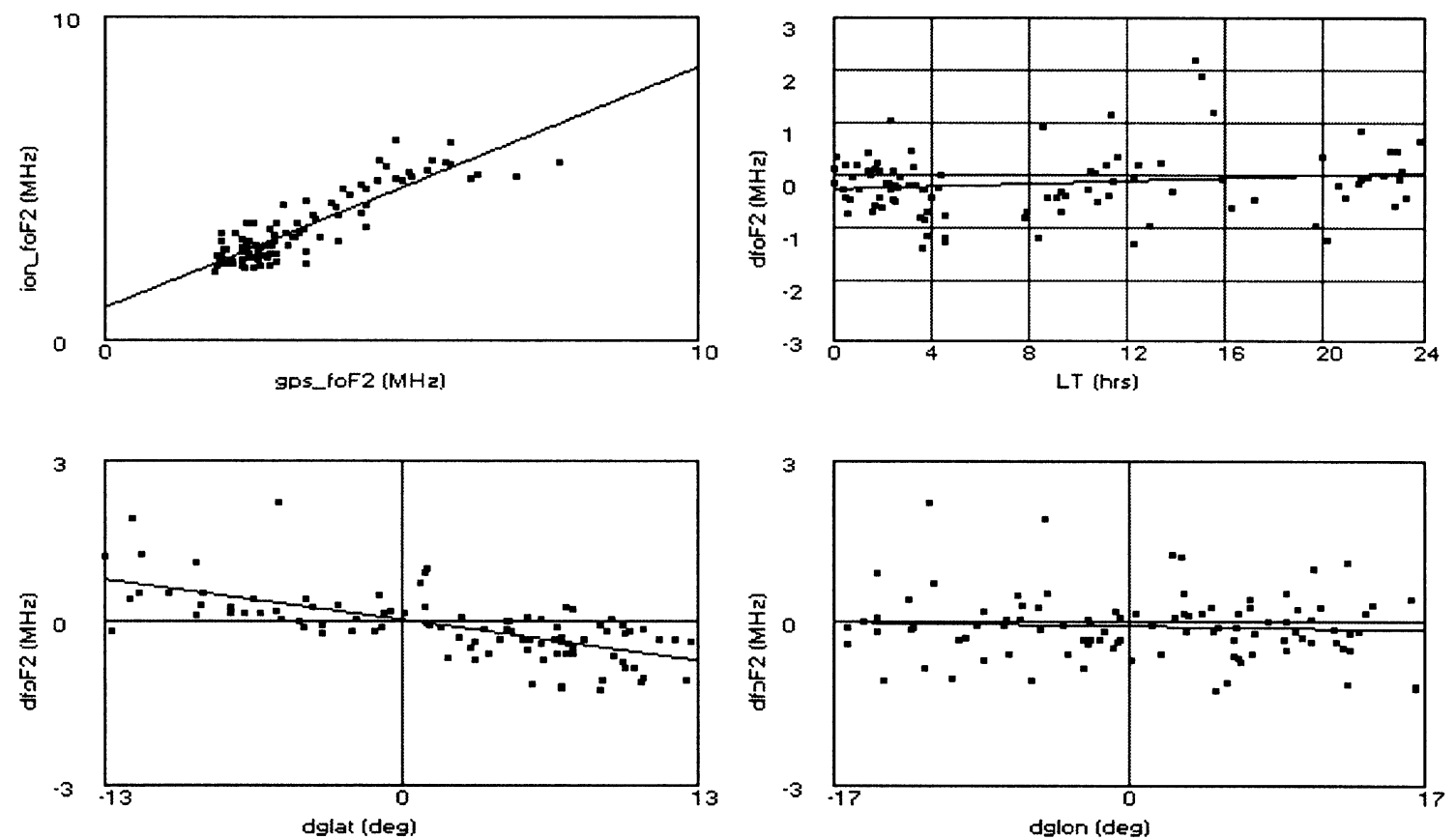

Fig. 7. The left-upper panel illustrates a scatterplot of $f_{o} F_{2}$ observed from GPS/MET occultation measurements and ionosonde measurements above Bear-Lake $\left(41.9^{\circ} \mathrm{N}, 111.4^{\circ} \mathrm{W}\right)$, Utah. The right-upper panel, left-lower panel, and right-lower panels illustrate scatterplots of the associated $f_{o} F_{2}$ differences in functions of local time, latitude difference, and longitude difference, respectively. The associated linear correlative coefficients are 0.88 (left-upper panel), 0.18 (right-upper panel), -0.67 (left-lower panel), and -0.05 (right-lower panel).

that the Chung-Li site $\left(24.6^{\circ} \mathrm{N}\right)$ is close to the latitude having electron density maximum in a plane within $\sim 20^{\circ} \mathrm{N} \pm 20^{\circ}$ latitude of the equatorial anomaly region. The observed result of under-estimated $f_{o} F_{2}$ by the Abel transform as shown in the right-upper panel of Fig. 6 is also matched to the diurnal development of the equatorial anomaly in early studies. Meanwhile, the angle between the occulting LEO and the corresponding auxiliary LEO position vectors is about $40^{\circ}$ 


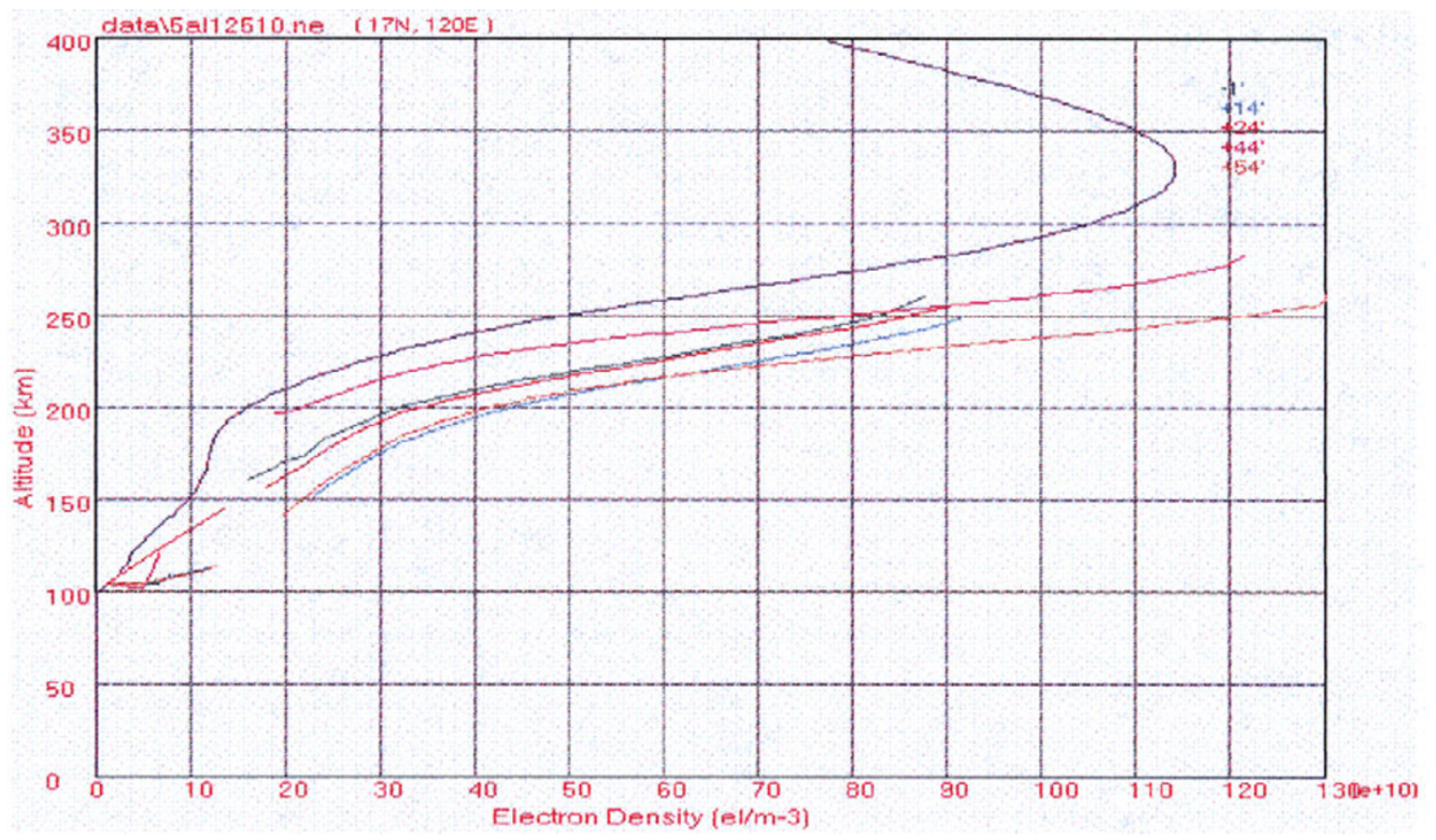

Fig. 8. Comparisons of ionospheric plasma density profiles obtained by GPS/MET occultation measurements (shown in blue color) using Abel inversion through TEC and from true-height analysis for ionograms (shown in other colors) recorded by the Chung-Li digisonde at $\sim 9: 25$ AM (local time) on October 21, 1995. The upper right-hand corner shows the ionogram recording time differences in minute to the referred occultation measurement.

for occultation observation at the $F_{2}$ peak. Therefore, due to the effect of the spherical symmetry assumption utilized for the Abel inversion, such even asymmetry would likely cause the Abel inversion to systematically under-estimate the electron density and even $f_{o} F_{2}$ at the tangent points near the peak densities of the equatorial anomaly.

For comparison with the mid-latitude dynasonde at BearLake, Utah, we collected 97 matches out of 100 occultations from 43 days (January 22 to February 11 and March 16 to April 6 in 1996) and where the location of the tangent point at $F_{2}$ peak is within \pm 17 degrees of the dynasonde site $\left(41.9^{\circ} \mathrm{N}\right.$, $\left.111.4^{\circ} \mathrm{W}\right)$ in latitude and longitude. During the days including daytime and nighttime, the Bear-Lake dynasonde was operating normally at a sounding interval of 5 minutes (a few ionograms were recorded at a 15 minute sounding rate); a high percentage of $97 \%$ has been approached in ionogram scaling analyses. Figure 7 shows the corresponding statistical comparison of derived $f_{o} F_{2}$ from the GPS/MET experiment and the dynasonde measurements in a similar manner as was done in Fig. 6 for the comparison with the Chung-Li digisonde data. In the left-upper panel of Fig. 7, the resulting linear correlation coefficient is 0.88 for the $f_{o} F_{2}$ values derived by GPS/MET occultation observations and the dynasonde measurements, which is close to the result $(0.85)$ obtained for the comparison with the Chung-Li digisonde. However, the corresponding $r m s f_{o} F_{2}$ difference is $0.61 \mathrm{MHz}$ or $16.5 \%$ in a fractional sense, which is much less than the previous comparison. The smaller $r m s \quad f_{o} F_{2}$ difference for the dynasonde site may be due to (1) smaller errors induced by the Abel inversion because the spherically symmetry assumption works better in the mid-latitude ionosphere and (2) better ionograms provided from the Bear-Lake dynasonde.
The other linear correlation coefficients are 0.18 for the corresponding $f_{o} F_{2}$ differences versus the local time (right-upper panel), -0.67 for the $f_{o} F_{2}$ differences versus the latitude differences (left-lower panel), and -0.05 for the $f_{o} F_{2}$ differences versus the longitude differences (right-lower panel). Similar to the results of Fig. 6, there is not much association of the $f_{o} F_{2}$ difference versus either the local time or the longitude difference. However the correlation coefficient value $(-0.67)$ for the scatterplot of the $f_{o} F_{2}$ difference versus the latitude difference denotes much more negative association compared to the correlative coefficient $(-0.20)$ obtained from the low-latitude Chung-Li digisonde data. And the corresponding least-squares fitting line for the scatterplot of the $f_{o} F_{2}$ difference versus the latitude difference represents that the critical frequency at $F_{2}$ peak is decreased $0.06 \mathrm{MHz}$ per degree increased in latitude. This result could be explained by that odd asymmetries in electron density exist at middle latitude, but the induced errors will tend to cancel at the Abel inversion utilizing the spherical symmetry assumption. Even asymmetries in electron density at the Chung-Li digisonde site, however, will cause larger errors of under-estimating near the peak densities of the equatorial anomaly.

For comparisons of derived electron density profiles, as mentioned above, analysis of ionograms from ground-based ionosonde measurements gives the variation of electron density with height up to the level of maximum density at the $F_{2}$ peak only. However, there are usually no coherent reflections from the starting range and valley in the ionosphere as shown as the ionogram derived profiles in Figs. 8 and 9; ground-based ionosondes have difficulties to observe these regions. On the other hand, GPS/MET occultation observations can retrieve ionospheric electron density through the 

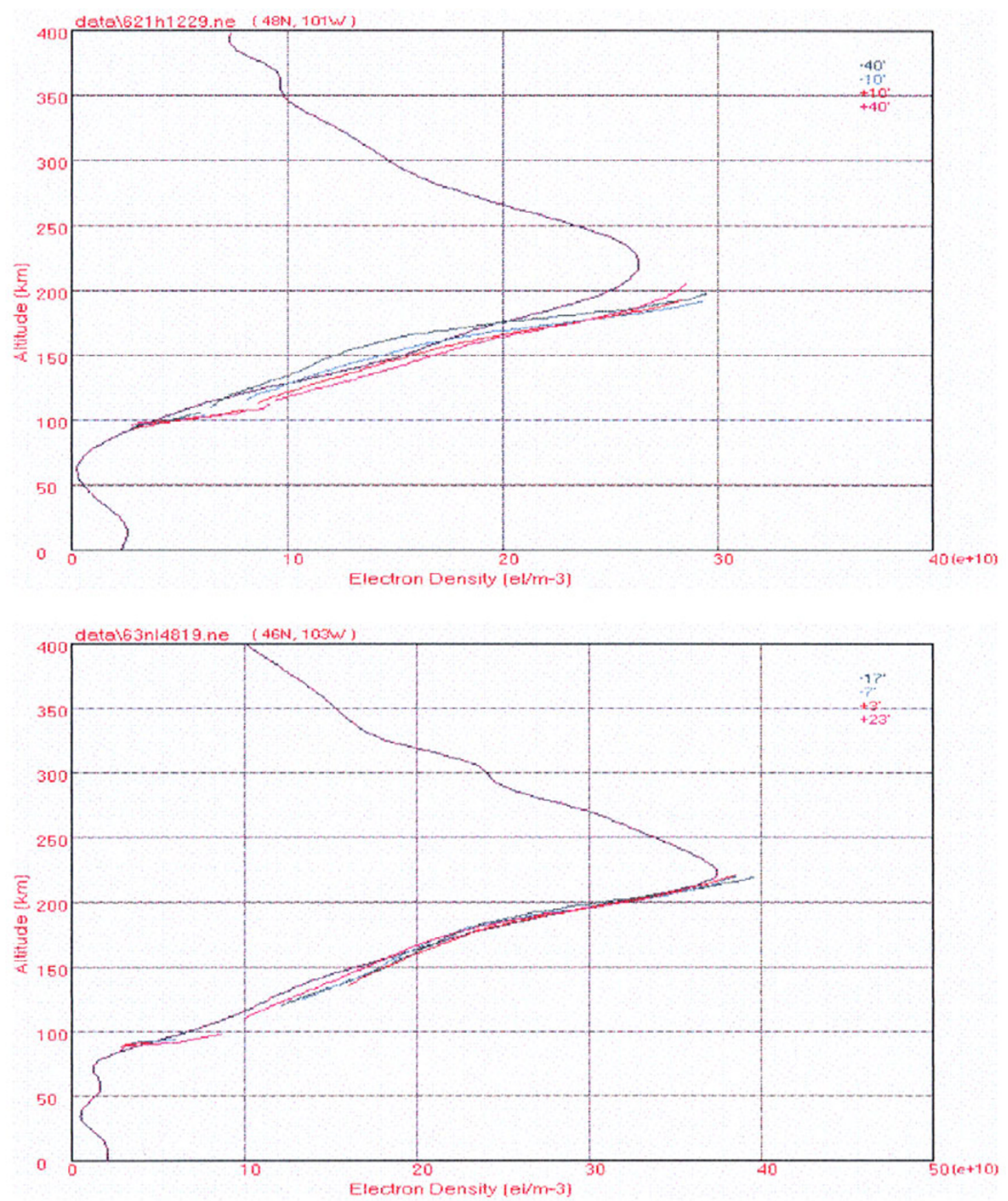

Fig. 9. Comparisons of ionospheric plasma density profiles obtained by GPS/MET occultation measurements (shown in blue color) using Abel inversion through TEC and from true-height analysis for ionograms (shown in other colors) recorded by the Bear-Lake Dynasonde at $\sim 11: 12$ AM LT on February 1 (the upper panel) and 3:48 PM LT on March 23 (the lower panel) in 1996. The upper right-hand corner shows the ionogram recording time differences in minute to the referred occultation measurement.

Abel inversion from the Earth surface to the LEO satellite altitude. Using the Abel transform to calibrated TEC profiles, Fig. 8 show plots of electron density profiles acquired at $\sim 0125$ UT $(0925$ LT) on the October 21, 1995 to compare the results obtained from GPS/MET data and ionograms recorded by the Chung-Li digisonde $\left(24.6^{\circ} \mathrm{N}, 121.0^{\circ} \mathrm{E}\right)$. The agreement is not so good; the corresponding error may be due to (1) asymmetry structure in equatorial region for the occultation reconstruction, (2) true-height analysis error caused by the valley problem and bad resolution for virtual range and sounding frequency of the Chung-Li digisonde, and (3) spatial mismatch of $6^{\circ}$ north to the ionosonde site for the top tangent point of the occultation measurement. It is also noted that the retrieved profile from GPS/MET data depicts a negative electron density below the altitude of $\sim 100 \mathrm{~km}$, which is physical impossibility and not shown. Such error is believed from spherical inhomogeneity of electron density and is happened more frequently for low-latitude region than mid-latitude region. In Fig. 9, another two examples show much better agreement of electron density profiles obtained from GPS/MET data and ionograms recorded by the BearLake dynasonde $\left(41.9^{\circ} \mathrm{N}, 111.4^{\circ} \mathrm{W}\right)$ on two different days of 
the first of February and the March 23, 1996. Meanwhile, there is observed a wavy structure at the topside electron density profiles in Fig. 9. It is difficult to justify such structure to be a real phenomenon or an error. Further validation using topside ionograms (the ISR electron density profiles having no enough accuracy) is necessary. However, we note that a wavy structure of electron density profile could be produced because of a cubic spline fitting to observed excess phases; the corresponding half wavelength can be estimated by the vertical component of an orbit trace between two contiguous GPS carrier phase change recordings which is close to $0 \mathrm{~km}$ at top of occultation and about $25 \mathrm{~km}$ at the $F_{2}$ peak (at 0.1 $\mathrm{Hz}$ recording rate).

\section{Conclusions and Future Prospects}

In this paper, we briefly describe the inversion techniques of the Abel integral transform for occultation observations and true-height analysis for ionograms. In practice, the main error of the Abel inversion is caused by the spherical symmetry assumption. On the other hand, the true-height analysis error is mainly caused by virtual-height measurement precision, starting-height error, $E F$ valley modeling error, and the error induced by gaps in virtual height information because of large sounding frequency interval, strong ionospheric absorption, or signal interference. The agreement of the results of the $F_{2}$-layer critical frequency and electron density profiles is better at mid-latitude observations than at low-latitude observations. The results also show that under-estimate of Chung-Li site $f_{o} F_{2}$ derived by the Abel transform is frequently occurred during afternoon and early evening. The reason for this could be due to spherically even asymmetric structure in the north equatorial anomaly region because of a fountain effect of vertical electrodynamic drift at the equator and plasma diffusion along geomagnetic field lines. Further studies to improve the Abel inversion technique or to develop other analysis methods, e.g. tomography, are necessary in the future.

Meanwhile, there are usually no coherent reflections from the starting range and valley in the ionosphere from ionograms; ground-based ionosondes have difficulties to observe these regions. Occultation observations can provide more information for the study of the lower part and valleys of the ionosphere in future research. Furthermore, in principle, GPS/MET occultation observations should provide more accurate electron density information above the $F_{2}$ peak than below since an integral technique such as the Abel transform starts used from the top altitude and proceeds downwards. But analysis of ionograms from ground-based ionosonde measurements gives the variation of electron density with height up to the level of maximum density at the $F_{2}$ peak only. Further topside ionospheric electron density validation needs to be done in the future using virtual height information from satellite-based ionosonde.

Acknowledgments. This work has been supported by Projects NSC89-2111-M008-047 and NSC88-NSPO(B)-RS3-FA07-02 (L.C. Tsai), NSC89-2111-M008-047 (W. H. Tsai), and NSF Grant OPP-9530935 to SDL/USU (F. T. Berkey).

\section{Appendix. The Abel Integral Transform}

The Abel integral equation is a typical case of Volterra integral equations of the first kind (Tricomi, 1985, Chapter 1). Vito Volterra (1860-1940) is the first to recognize the importance of the theory of integral equations and to consider it systematically. In fact, Abel solved the Abel integral equation already in 1825, and in essentially the same manner which we shall use; however, he did not realize the general importance of such types of functional equations. The important Abel integral equation can be expressed as

$$
\int_{0}^{x} K(x, y) \phi(y) d y=f(x),
$$

where the kernel

$$
K(x, y)= \begin{cases}\frac{1}{\sqrt{x-y}}, & \text { if } y \leq x \\ 0, & \text { if } y>x .\end{cases}
$$

To solve the Abel integral equation, the compositions of both side of Eq. (A.1) with the kernel $K(x, y)$ can be derived and give us

$$
\begin{aligned}
\int_{0}^{x} K(x, z)\left(\int_{0}^{z} K(z, y) \phi(y) d y\right) d z \\
\quad=\int_{0}^{x}\left(\int_{0}^{x} \frac{1}{\sqrt{x-z}} \frac{1}{\sqrt{z-y}} d z\right) \phi(y) d y \\
\quad=\int_{0}^{x} \frac{f(y)}{\sqrt{x-y}} d y .
\end{aligned}
$$

We define $K_{2}(x, y)$ to be the composition of $K(x, y)$ itself and use the substitution $z=y+(x-y) t$, which gives us

$$
\begin{aligned}
K_{2}(x, y) & =\int_{0}^{x} K(x, z) K(z, y) d z \\
& =\int_{0}^{x} \frac{1}{\sqrt{x-z}} \frac{1}{\sqrt{z-y}} d z \\
& =\int_{0}^{1}[t(1-t)]^{-1 / 2} d t=\pi .
\end{aligned}
$$

Hence, we have the amazingly simple solution for the Abel integral equation

$$
\phi(x)=\frac{1}{\pi} \frac{d}{d x} \int_{0}^{x} \frac{f(y)}{\sqrt{x-y}} d y .
$$

More explicitly; we can integrate by parts, and, provided the function $f$ is differentiable, we obtain

$$
\phi(x)=\frac{f(0)}{\pi \sqrt{x}}+\frac{1}{\pi} \int_{0}^{x} \frac{f^{\prime}(y)}{\sqrt{x-y}} d y .
$$

The Eqs. (A.1) and (A.2) are the corresponding Abel integral transform.

\section{References}

Argo, P. E. and M. Hindman, PC radar-A data acquisition tool, Presonal Engineering and Instrumentation News, 49-56, August 1987.

Bibl, K. and B. Reinisch, The universal digital ionosonde, Radio Sci., 13, 519, 1978.

Born, M. and E. Wolf, Principles of Optics, 808 pp., New York, Pergamon, 1980. 
Budden, K. G., The Propagation of Radio Waves, 669 pp., Cambridge University Press, Cambridge, 1985.

Fjeldbo, G. and V. R. Eshleman, Atmosphere of Venus as studied with the Mariner V dual radio frequency occultation experiment, Radio Sci., 4, 879-897, 1969.

Fjeldbo, G., A. J. Kliore, and V. L. Eshleman, The neutral atmosphere of Venus as studies with the Mariner V radio occultation experiments, Astron. J., 76, 123-140, 1971.

Grubb, R. N., The NOAA SEL HF radar system (ionospheric sounder), NOAA Tech. Memo. ERL SEL-55, Space Environ. Lab., Boulder, 1979.

Gulyaeva, T. L., W. Becker, L. F. McNamara, A. K. Paul, J. E. Titheridge, and J. W. Wright, Analysis of numerical ionograms: the starting problem, Ionospheric Prediction Service Series X Reports, IPS-X7, Aust. Gov. Dep. of Sci. and Technol., Ionos. Predict. Serv., Sydney, Australia, 1978.

Hajj, G. A. and L. J. Romans, Ionospheric electron density profiles obtained with the Global Positioning System: Results from the GPS/MET experiment, Radio Sci., 33(1), 175-190, 1998.

Hajj, G. A., L. C. Lee, X. Pi, L. J. Romans, W. S. Schreiner, P. R. Straus, and C. Wang, COSMIC GPS ionospheric sensing and space weather, Terrestial, Atmospheric and Oceanic Sciences, 11(1), 235-272, 2000.

Kliore, A. J., D. L. Cain, G. S. Levy, V. R. Eshlemann, G. Fjeldbo, and F. D. Drake, Occultation experiments: Results of the first direct measurements of Mars' atmosphere and ionosphere, Science, 149, 1243-1248, 1965.

Kursinski, E. R., G. A. Hajj, J. T. Schofield, R. P. Linfield, and K. R. Hardy, Observing Earth's atmosphere with radio occultation measurements using the Global Positioning System, J. Geophys. Res., 102, 23429-23465, 1997.

Lindal, G. F., H. B. Hotz, D. N. Sweetnam, Z. Shippony, J. P. Brenkle, G. V. Hartsell, R. T. Spear, and W. H. Michael, Viking radio occultation measurements of the atmosphere and topography of Mars: Data acquires during 1 Martian year of tracking, J. Geophys. Res., 84, 8443-8456, 1979.

Lindal, G. F. and Coauthors, The atmosphere of Jupiter: An analysis of the Voyager radio occultation measurements, J. Geophys. Res., 86, 8721$8727,1981$.

Lindal, G. F. and Coauthors, The atmosphere of Uranus: results of radio occultation measurements with Voyager 2, J. Geophys. Res., 92, 1498715001, 1987.

Martyn, D. F., The normal F region of the ionosphere, Proc. IRE N. Y., 47, 147,1959

McNamara, L. F. and J. E. Titheridge, Numerical ionograms for comparing methods of N(h) analysis, IPS Series R Reports X-5, 1977.

Paul, A. K. and D. L. Mackison, Scaling of the F-layer critical frequency from digital ionograms applied to observations during the solar eclipse on 26 February 1979, J. Atmos. Terr. Phys., 43(3), 221-223, 1981.

Rastogi, R. G., The diurnal development of the anomalous equatorial belt in the F2 region of the ionosphere, J. Geophys. Res., 64, 727, 1959.

Reinisch, B. W., New techniques in ground-based ionospheric sounding and studies, Radio Sci., 21, 331, 1986.

Rocken, C., R. Anthes, M. Exner, D. Hunt, S. Sokolovskiy, R. Ware, M. Gorbunov, W. Schreiner, D. Feng, B. Herman, Y. Kuo, and X. Zou, Analysis and validation of GPS/MET data in the neutral atmosphere, $J$. Geophys. Res., 102(D25), 29849-29866, 1997.

Schreiner, W. S., S. V. Sokolovskiy, C. Rocken, and D. C. Hunt, Analysis and validation of GPS/MET radio occultation data in the ionosphere, Radio Sci., 34(4), 949-966, 1999.

Titheridge, J. E., Ionogram analysis with the generalised program POLAN, World Data Center A for Solar-Terrestrial Physics, Report UAG-93, 1985.

Titheridge, J. E., Starting models for the real height analysis of ionograms, J. Atmos. Terr. Phys., 48, 435-446, 1986.

Titheridge, J. E., The real height analysis of ionograms: A generalized formulation, Radio Sci., 23, 831-849, 1988.

Tricomi, F. G., Integral Equations, 238 pp., Dover, Mineola, N. Y., 1985.

Tsai, L.-C., F. T. Berkey, and G. S. Stiles, The true-height analysis of ionograms using simplified numerical procedures, Radio Sci., 30(4), 949-959, 1995.

Tsai, L.-C. and F. T. Berkey, Ionogram analysis using fuzzy segmentation and connectedness techniques, Radio Sci., 35(5), 1173-1186, 2000.

Ware, R. and Coauthors, GPS sounding of the atmosphere from low Earth orbit: preliminary results, Bulletin of the American Met. Soc., 77, 19-40, 1996.

Wright, J. W. and M. L. V. Pitteway, Real-time data acquisition and interpretation capabilities of the Dynasonde, 1, Data acquisition and real-time display, Radio Sci., 14(5), 815-825, 1979.

$\mathrm{Wu}, \mathrm{J}$. T., Elimination of clock errors in a GPS based tracking system, paper AIAA-84-2052, AIAA/AAS Astrodynamics Conference, Seattle, WA, August, 1984.

Zou, X. Y., H. Kuo, and Y.-R. Guo, Assimilation of atmospheric radio refractivity using a nonhydrostatic mesoscale model, Mon. Wea. Rev., 123, 2229-2249, 1995.

L.-C. Tsai(e-mail: lctsai@csrsr.ncu.edu.tw), W. H. Tsai, W. S. Schreiner, F. T. Berkey, and J. Y. Liu 